\title{
El aprendizaje basado en problemas para la intervención de la Enfermería con la persona adulta mayor ${ }^{1}$
}

Universidad de Costa Rica

\author{
Ensayo
}

Escuela de Enfermería

Elena Mora Escalante ${ }^{2}$

\section{COMO CITAR}

Mora Escalante, Elena. El aprendizaje basado en problemas para la intervención de Enfermería con la persona adulta mayor. Rev. Enfermería Actual en Costa Rica [en línea].2011, No.20 [citado (fecha)]. Disponible World Wide Web:

$<$ http://www.revenf.ucr.ac.cr/mayor.pdf> ISSN 1409-4568

\section{RESUMEN}

El aprendizaje basado en problemas se origina en universidades como Case Western Reserve, en los Estados Unidos, la Universidad de McMaster, en Canadá, de modo que otros centros universitarios en el mundo lo han integrado como metodología de enseñanza y aprendizaje en diversas áreas del conocimiento y en distintas carreras. Por lo anterior, la Universidad de Costa Rica, a través del Departamento de Docencia Universitaria, elaboró el curso "Aprendizaje Basado en Problemas" dirigido a las y los profesores con el fin de desarrollar competencias docentes para su utilización. Luego de haber participado en dicho curso, nace la inquietud de realizar el presente ensayo para reflexionar sobre el ABP y su aplicación como técnica didáctica en el Módulo Intervención de Enfermería con la Adultez Mayor, la cual pertenece a la carrera de Enfermería de la Universidad de Costa Rica. El ABP es una estrategia de enseñanza - aprendizaje en la que la adquisición del conocimiento y el desarrollo de habilidades y actitudes tienen la misma importancia debido que buscan facilitar la formación del estudiante y desarrollar un razonamiento y el juicio crítico que le permitan enfrentarse a los desafíos de la vida profesional, todos aspectos relevantes que se contemplan en el módulo.

PALABRAS CLAVE: Aprendizaje-Basado-Problemas, Enfermería, Persona-adulta-mayor.

\footnotetext{
${ }_{2}^{1}$ Fecha de recepción: 2 de febrero del 20100

Fecha de aceptación: 15 de marzo del 2011

${ }^{2}$ Profesora Adjunta de la Escuela de Enfermería de la Uni versidad de Costa Rica. Enfermera especialista en Salud Mental y Psiquiatría. Master en Terapia Familiar Sistémica. E mail: moresca21@ @otmail.com
} 


\title{
Problem-based learning and its application in nursing intervention with the elderly person
}

\begin{abstract}
Essay
University of Costa Rica

School of Nursing

\section{Cited}

Mora Escalante, Elena. Problem-based learning and its application in nursing intervention with the elderly person. Rev. Enfermería Actual en Costa Rica [en línea].2011, No.20 [citado (fecha)]. Disponible World Wide Web:

<http://www.revenf.ucr.ac.cr/mayor.pdf> ISSN 1409-4568

ABSTRACT

Learning based on problems (LBP) arises from the Case Western Reserve University in the United States and McMaster University in Canada and universities worldwide have integrated it as a methodology of teaching and learning in different areas of knowledge and in different races. Therefore, the University of Costa Rica through the University Teaching Department, developed the course Learning Based on Problems and aimed at teachers to develop teaching skills for use. After participating in this course, comes the concern for the present essay, to reflect on the LBP and its application as a teaching technique in the Module Nursing Intervention with the older adult, part of the undergraduate course at the University of Costa Rica. LBP is a teaching strategy - learning, where knowledge acquisition and development of skills and attitudes are equally important, designed to facilitate student training and to de velop reasoning and critical thinking, enabling it to cope with challenges of working life, any areas that are covered in the course.
\end{abstract}

Elena Mora Cascante

KEY WORDS: Adult-major-person, Learning-Based-Problems, Nursing.

\section{INTRODUCCIÓN}

El Módulo intervención de Enfermería con la adultez mayor, impartido en la Escuela de Enfermería de la Universidad de Costa Rica, está diseñado para que el estudiantado adquiera conocimientos que le permitan brindar atención integral a las personas adultas mayores en los distintos escenarios, según las necesidades detectadas. (Módulo intervención de enfermería con la adultez mayor, 2010)

Para lograr este propósito, en este módulo se ha generado un gran interés por enriquecer la práctica docente mediante nuevas formas de enseñar y aprender, razón que obliga a utilizar diferentes metodologías que favorecen el aprendizaje activo del estudiante y la integración de la teoría con la práctica. Dichas metodologías son: el 


\section{Revista Electrónica Entomeria actual en costa Rica}

cineforum, el videoforum, los sociodramas, la reconstrucción de relatos, la mesa redonda, las revisiones bibliográficas, las discusiones grupales, los talleres, el trabajo de campo, la observación dirigida y las discusiones de casos.

Dichas metodologías están centradas en el estudiante, ya que le ayudan a construir el conocimiento con base en sus habilidades de modo que el alumnado es responsable de edificar de su conocimiento, modelo contrario a las actuales metodologías centradas en la comunicación de saberes por parte del profesorado, quien ejerce la función de transmisor de la información y en el que el estudiante sigue siendo sujeto pasivo. Cabe recordar que los contenidos aprendidos de memoria tienden a olvidarse rápidamente y la estructura cognitiva del aprendiz no es mejorada o modificada para aclarar ideas erróneas, por tanto, los errores de concepto adquiridos persistirán y el potencial del conocimiento aprendido para uso futuro o solución de problemas es poco o nulo (Novak, 1983).

Dado lo anterior, el propósito del presente ensayo es reflexionar acerca de utilizar el Aprendizaje Basado en Problemas (ABP) como una técnica didáctica en el módulo Intervención de Enfermería con la Adultez Mayor, ya que es una estrategia de enseñanza - aprendizaje en la que la adquisición del conocimiento y el desarrollo de habilidades y actitudes tienen la misma importancia, aspectos relevantes que se contemplan en el módulo. El ABP es un enfoque pedagógico multi-metodológico y multi-didáctico encaminado a facilitar el proceso de enseñanzaaprendizaje y de formación del estudiante, además de que desarrolla el razonamiento y el juicio crítico en el estudiantado (Castillo, V. 2010).

\section{EL APRENDIZAJE BASADO EN PROBLEMAS}

En el año 1960 inició un estilo de enseñanza centrado en el estudiante y que tiene como una herramienta metodológica fundamental al Aprendizaje Basado en Problemas (ABP), el cual fue aplicado en la Facultad de Medicina de la Universidad de McMaster (Ontario, Canadá). Este tipo de enseñanza es el predilecto en muchas facultades de medicina alrededor del mundo, debido a que el ABP promueve una participación más activa del estudiante en su propia educación, transformación que mejora el proceso del aprendizaje. Asimismo, se ha demostrado que el estudiantado prefiere este sistema, porque estimula las actividades de autoaprendizaje al utilizar un número mayor de fuentes de información. (Cáceres \& otros, 2010)

A través del ABP, el y la estudiante "aprende a aprender" y desarrolla un pensamiento crítico y reflexivo apoyado en el autoaprendizaje (Guillamet, L \& otros, 2010). Su sustento son las diferentes teorías del aprendizaje humano, en particular, la teoría constructivista que considera que el conocimiento es el resultado de un proceso de construcción en el que la persona participa de forma activa. Para Ausubel (2002), la educación apoyada en el constructivismo, implica experimentación y la resolución de problemas.

Según Molina, J. \& otros (2001), en su artículo Aprendizaje basado en problemas: una alternativa al método tradicional, el ABP enseña los contenidos al estudiante basándose en casos, lo cual le permite la observación y análisis de actitudes y valores que, durante el método tradicional docente, no pueden llevarse a cabo.

El ABP es una propuesta didáctica basada en la idea de hacer que las y los estudiantes no se limiten a escuchar para aprender, sino que solucionen problemas. Entonces, no consiste en contarles la información como si se 


\section{Revista Electrónica Entomeria actual en costa Rica}

estuviera siendo leída de un libro, por el contrario, promover que ellos y ellas se enfrenten a la información a través de un problema con el que está relacionada. Esto provoca en el cerebro una serie de reacciones al utilizar todos sus recursos cognitivos, ya que no se podemos seguir "anclados(as)" en los contenidos, dado que debe dársele a estos el lugar que merecen: el de medios y de recursos. La enseñanza de habilidades y procesos de pensamiento como contenido fundamental se debe asumir con valentía, .por lo que "La finalidad máxima de la formación específica del ser humano no es la comprensión del mundo que lo rodea, sino el desarrollo de aquellas habilidades que le permiten adaptarse y adaptar la realidad de una manera significativa” (De Zubiría. S. Miguel. 2004)

Entre las características más destacables del ABP, Bernabeu (2004) menciona los siguientes:

- Fomenta la actitud positiva hacia el aprendizaje.

- Facilita no sólo la adquisición de conocimientos de la materia, sino que ayuda al estudiante a crear una actitud favorable para el trabajo en equipo, acontecimiento imprescindible en la formación de profesionales en Enfermería para quienes la realidad laboral y docente se basa en el trabajo en equipo.

- Existe un respeto por la autonomía del estudiante.

- Es un método de trabajo activo con intensa participación del estudiante.

- Está orientado a la solución de problemas que conducen a la consecución de aprendizajes.

- Se centra en el estudiante y no en el profesor o en los contenidos.

- El rol del docente es ser un facilitador del aprendizaje.

Por otra parte, si el ABP es considerado una técnica didáctica su nombre cambia a método de caso, el cual tiene por objeto acercar una realidad concreta a una serie de personas que se proponen trabajar en un determinado ámbito. Lo más importante del método es que permi te que los problemas se estudien en toda su complejidad, incluyendo elementos de la experiencia que serían muy difícil o quizás imposible reproducir en el marco de una clase (Pacheco, G.L. 2010).

Como lo menciona Riverón, P. \& otros. (2000), el modelo ABP utiliza situaciones problemáticas para conducir el aprendizaje y puede concretarse en un proyecto de investigación, en un método de estudio de casos, en un proyecto de diseño, etc. Lo realmente importante no es como se le nombre, sino considerar que el ABP es una alternativa distinta del método tradicional, opuesta a la estrategia expositiva o magistral; utilizado como técnica permite profundizar sobre un problema, tratando de comprender una situación específica y singular, además, dota al estudiante de estrategias de aprendizaje que le permitirán afrontar las nuevas necesidades para enfrentarse a los retos de la vida profesional.

Precisamente, este es un objetivo que persigue alcanzar el Módulo de intervención de Enfermería con la adultez mayor a través del estudio de caso y que se puede lograr mediante un cambio didáctico, es decir, al utilizar el ABP. Por una parte, conocer las condiciones reales y particulares de vida de una persona adulta mayor, reconocer sus necesidades y proponer en forma conjunta alternativas de solución y, por otra, promover la utilización efectiva del conocimiento en contextos significativos, lo cual considera el desarrollo de habilidades como eje fundamental. Lo anterior se consigue mediante el trabajo conjunto del facilitador(a) y el/la estudiante, en un espacio donde ambos descubren las estrategias apropiadas para la adquisición del conocimiento tanto en lo referente a lo teórico como a lo práctico, contrario al modelo tradicional en el cual el acto educativo se centra en los contenidos de 
aprendizaje entendido como temas o información acumulada. Por su parte, la preocupación fundamental del ABP es que el/la estudiante aprenda, es decir, que aprenda a desempeñarse en contextos con sentido propio. (De Zubiría, 2004)

Existen diversos métodos para poner en práctica el Aprendizaje Basado en Problemas, sin embargo, en el presente ensayo se considerará el Método de las Cinco Fases que consiste en:

1) Asignación del problema o caso. Didácticamente, los problemas que el/la docente selecciona para incluir en la experiencia del ABP deben basarse en el criterio de significatividad, idea que implica dos aspectos: a) que sean los problemas con los que efectivamente se enfrentará el y la estudiante en la realidad y b) que sean problemas que puedan resolverse en el entorno de discusión del aula real y concreta.

En el módulo de intervención de enfermería con la adultez mayor, el problema o caso por elegir, ya sea en el Centro Hospitalario o en el Hogar de Ancianos, es la persona adulta mayor y sus cambios morfológicos, fisiológicos, psicológicos y sociales que enfrenta en su proceso de envejecimiento en el que se contemplan los siguientes aspectos: Datos personales, situación socioeconómica, relaciones familiares, actividades sociales, antecedentes patológicos personales y familiares, hábitos personales de higiene, alimentación, evacuaciones intestinales, eliminaciones vesicales y patrón de sueño. Además, su salud sexual y reproductiva, salud mental, farmacoterapia, aspectos espirituales, aspectos relacionados son su visión de género, capacidades funcionales y factores de riesgo social.

Para los fines académicos, el objetivo final no es la resolución del problema. El problema se utiliza como sustento de la identificación de los temas de aprendizaje para su estudio, ya sea de manera independiente o grupal, en cuyo abordaje se pueden utilizar materiales como lecturas, audio y videos como generadores de polémica (Molina \& otros. 2003).

2) Diseño de alternativas de solución o generación de soluciones posibles. Primeramente, se realiza una lluvia de ideas y se genera una hipótesis para revisar qué se sabe de la situación o lo que creen saber acerca del caso, poniendo en práctica todo el conocimiento previo que poseen en relación con los diversos temas, pasos que permiten analizar los conocimientos previos que poseen las y los estudiantes que, como hemos visto a través de las teorías constructivistas, son imprescindible para la construcción de nuevos conocimientos.

En la intervención de Enfermería con la adultez mayor se analizan los elementos teóricos relacionados con el problema y se elaboran propuestas y estrategias concretas de intervención, de carácter único, irrepetible y peculiar para cada caso.

3) Identificar los objetivos de aprendizaje. En el módulo de intervención de enfermería con la adultez mayor, el objetivo es: Analizar las desviaciones de la salud en las personas adultas mayores y la repercusión de estos eventos en el ámbito personal, familiar y comunitario, considerando los aspectos físicos, socioculturales y espirituales desde la perspectiva de género sensitiva y brindar una atención de enfermería libre de mitos y estereotipos, reconociendo y respetando las particularidades tanto en el aspecto biológico como psicosocial y según las necesidades y problemas propios de la persona adulta mayor. 
4) Realizar una lectura e investigación individual para preparar la plenaria final. Se debe organizar un plan de búsqueda de las fuentes de información que permita adquirir el conocimiento necesario y llevarla a cabo.

5) Hacer una plenaria final de grupo y entrega del informe final. En este punto se debe sintetizar la información y discutir los principios y conceptos aprendidos de este escenario / problema, además, se debe realizar un informe final de las soluciones propuestas al problema.

Es de vital importancia elaborar un cronograma de trabajo para desarrollar las diferentes etapas, ya que conlleva discusiones grupales y espacios de reflexión y diálogo de forma frecuente. Este cronograma lo complementa el grupo de estudiantes con una distribución de tareas específicas para los miembros.

En resumen, De Zubiría (2004) puntualiza que para el desarrollo de esta experiencia, se requiere de la inmersión de las y los estudiantes en el problema, la identificación de conocimientos poseídos e ignorados sobre el problema, la definición y estructuración del problema, la reunión y distribución de la información sobre el problema, la generación de soluciones, la evaluación de las mejores soluciones, la adopción de las soluciones y la realización de un informe final de la(s) solución(es) propuesta(s).

Es importante mencionar que para poner en marcha esta técnica, además se requiere que el grupo de estudiantes o grupo tutorial trabaje de manera colaborativa, es decir, intercambiando ideas y materiales tanto entre profesorado y alumnado como entre los mismos estudiantes, por otra parte, se debe desarrollar en grupos pequeños compuesto de 6 a 10 estudiantes- y contar con un tutor(a). El/la profesor(a) debe ser un creador(a), un guía que estimule a los y las estudiantes a aprender, a descubrir y sentirse satisfechos (as) por el saber acumulado, lo cual puede lograrse si aplica correctamente el ABP. (Riverón, P. \& otros. 2000)

La evaluación en el ABP se da a lo largo del proceso y está conformado por tres variables: a) evaluar los conocimientos adquiridos, b) evaluar la dinámica de participación de las y los estudiantes del grupo y c) evaluar la propuesta de solución escogida. Para este proceso evaluativo, se puede valorar la utilización de diferentes estrategias tales como examen práctico, mapas conceptuales, autoevaluaciones, presentación oral, memoria, reporte, entre otros aspectos; para visualizar el logro de los objetivos.

\section{CONCLUSIONES}

Se puede afirmar que el $\mathrm{ABP}$ es una forma de trabajo que constituye una alternativa valiosa para el método tradicional de aprendizaje, ya que el estudiantado pasa a ser un sujeto activo dentro de su formación, puesto que es él o ella quien busca el aprendizaje que considera necesario para la resolución de los problemas que se le plantean. Por lo anterior, aumenta su capacidad para el autoaprendizaje y su capacidad crítica para analizar la información que les ofrece la búsqueda.

El objetivo final no es la resolución del problema. El problema se utiliza como sustento de la identificación de los temas de aprendizaje para su estudio por lo que se debe recordar que lo importante es el aprendizaje y no la resolución del problema si no ¿para qué se evalúa? y ¿qué se evalúa? 
A través de la utilización del ABP, se crean espacios de narración de experiencias que brindan al estudiantado la oportunidad de expresar sus ideas y opiniones y, al docente, de realizar un cambio en su mentalidad y asumir un rol de tutor(a) afiliativo y no un rol directivo en el proceso. Es verse a sí como un guía del proceso de otros seres humanos que necesitan mirar hacia atrás para construir sus propias respuestas, por tanto, lo ayuda a solucionar dudas y, primordialmente, a plantear buenas preguntas. El tutor es el lazarillo del estudiante en su proceso de aprendizaje.

Ortiz y Otros, profesores de la EU Enfermería de la Comunidad de Madrid (2003), en Aprendizaje basado en problemas: una alternativa al método tradicional, señalan que es interesante mencionar una serie de claroscuros que en el transcurso de los siete años de experiencia han podido detectar: a) A lo largo del tiempo, las y los protagonistas del ABP han ido cambiando, mejorando en habilidades y conocimiento de la metodología, pero también por ello han logrado ser más críticos respecto de las partes más débiles del sistema. b) El estudiantado reconoce las ventajas del método, porque aumenta su capacidad para el autoaprendizaje y su capacidad crítica para analizar la información que les ofrece la búsqueda. Sin embargo, encuentran dificultades con el método, porque al convertirse en elementos activos de su aprendizaje se ven impelidos a dedicar más tiempo a estas asignaturas y finalmente c) Se muestran inseguros(as) acerca de la suficiencia del conocimiento adquirido por este método.

Este modelo no es simple a pesar de que sus principios orientadores son fácilmente comprensibles. Por lo tanto, para utilizarlo en la Escuela de Enfermería de la Universidad de Costa Rica y apoyar el aprendizaje activo del estudiante por medio de este método, es imprescindible que se inicie progresivamente, invirtiendo en la capacitación de su recurso humano, dado que el ABP exige un cambio en la práctica docente.

\section{BIBLIOGRAFIA}

Ausubel, D. P. (2002). Adqui si ción y retención del conocimiento. Una perspectiva cognitiva. Barcelona: Editorial.

Bernabeu, D. (2004). El aprendizaje Basado en Problemas: Una herramienta para toda la vida. Escuela de Enfermería de Vall d'Hebron, en Agencia Lain Entralgo. Obtenido el 2 de noviembre de 2010 desde http://www.cedus.cl/files/DOCENCIA\%20UNIVERSITARIA\%20ABP.pdf

Bernabeu, $\mathrm{M}^{\mathrm{a}}$; Consul, M.(2004). Similitudes entre el proceso de convergencia en el ámbito de la Educación Superior Europea y la adopción del Aprendizaje Basado en Problemas en la E.U.I Vall d'Hebron de Barcelona. Revista Interuniversitaria de Formación del Profesorado. 18 (1), 5.

Castillo, V. (2010). El Aprendizaje Basado en Problemas como un enfoque pedagógico en la educación en salud. Revista Colombia Médica, 32 (004), 189-196. Obtenido el 10 de noviembre de 2010 desde http://redalyc.uaemex.mx/redalyc/pdf/283/28332407.pdf

Cáceres \& otros. (2010). Aprendizaje Basado en Problemas. Obtenido el 2 de diciembre de 2010 desde http://www2.uah.es/problembasedlearning/formacion\%20del\%20profesorado.htm

De Zubiría. S. (2004). Enfoques Pedagógicos y Didácticas Contemporáneas. Bogotá: Editorial Transversal.

Guillamet, L. \& otros. (2010). Aprendi zaje Basado en Problemas. Estrategia de Implantación. Escuela Universitaria de Enfermería Virgen de las Nieves. Adscrita a la Universidad de Granada. Obtenido el 12 de noviembre de 2010 desde http://www.erol.es/articu/anterior/09_02.php?num=32(02) 


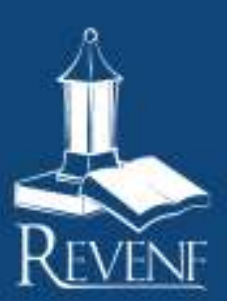

\section{Revista Electrónica Enfermeria Actual en costa Rica}

Modulo intervención de enfermería con la adultez mayor. (2010). Programa del curso. Universidad de Costa Rica.

Molina J., García, A., Pedraz, M.. (2003). Aprendizaje basado en problemas: una alternativa método tradicional . Revista de la Red Estatal de Docencia Universitaria. 3 (2), 5.

Novak, Anexión. (1983). Psicología educativa: un punto de vista cognoscitivo. México, D.F.: Editorial Trillas.

Pacheco, L. (2010). Aprendizaje Basado en Problemas. Departamento de Docencia Universitaria. Universidad de Costa Rica.

Riverón, O. \& otros. (2000). El Aprendizaje Basado en Problemas: Una alternativa Educativa. Revista Digital de Educación y Nuevas Tecnologías, 18. Obtenido el 27 de noviembre de 2010 desde http://www.scribd.com/doc/21679558/Aprendizaje-basado-enproblemas-y-metodo-de-proyectos 